\title{
Minkowski Uzayında Asli Regle Yüzeylerin Kesit Ĕgriliği
}

\author{
Soley ERSOY*(D), Murat TOSUND
}

Sakarya Üniversitesi, Matematik Bölümü, Sakarya, TÜRKIYYE

Geliş / Received: 19/09/2019, Kabul / Accepted: 21/02/2020

\begin{abstract}
$\ddot{O} z$
Bu çalışmada, $n$-boyutlu Minkowski uzayında timelike doğrultman uzaylı merkez regle yüzeyli genelleştirilmiş timelike regle yüzeyin dayanak eğrisinin merkez noktalarında verilen asli ışınların dayanak eğrisi boyunca hareketiyle oluşan 2-boyutlu asli regle yüzeyler göz önüne alınmıştır. Böylece 2-boyutlu timelike asli regle yüzeyinin kesit eğriliği ile asli dağılma parametresi arasındaki bağıntı elde edilmiş ve bu bağıntının 3-boyutlu Minkowski uzayındaki bir timelike regle yüzeyin Gauss eğriliği ve dağılma parametresi arasındaki bağıntının genelleştirilmişi olduğu görülmüştür. Benzer şekilde spacelike asli regle yüzeyinin kesit eğriliği ile asli dağılma parametresi arasındaki bağıntı elde edilmiştir. Bu bağıntının da 3-boyutlu Minkowski uzayındaki bir spacelike regle yüzeyin Gauss eğriliği ve dağılma parametresi arasındaki bağıntının genelleştirilmişi olduğu belirlenmiştir.
\end{abstract}

Anahtar Kelimeler: Regle yüzey, kesit eğriliği, dağılma parametresi

\section{Sectional Curvature of Principal Ruled Surfaces in Minkowski Space}

\begin{abstract}
In this study, 2-dimensional principal ruled surfaces obtained by the motion of the principal rays, given at the central points of the base curve of a generalized timelike ruled surface with timelike generating space and central ruled surface in $n$-dimensional Minkowski space, throughout the base curve has been considered. In this way, the relationship between the sectional curvature and principal distribution parameter of 2-dimensional timelike principal surface has been given. It is found that this relationship is a generalization of the relationship between the Gaussian curvature and distribution parameter of a timelike ruled surface in 3-dimensional Minkowski space. In a similar way, the relationship between the sectional curvature and principal distribution parameter of the spacelike principal surface has been obtained. This relationship is a generalization of the relationship between the Gaussian curvature and distribution parameter of a spacelike ruled surface in 3-dimensional Minkowski space.
\end{abstract}

Keywords: Ruled surface, sectional curvature, distribution parameter

\section{Giriș}

2-boyutlu bir yüzeyin Gauss eğriliği ile dağılma parametresi arasındaki bağıntı (Kruppa, 1957) tarafindan verilmiştir ve bu bağıntı klasik yüzey teorisinde Lamarle formülü olarak adlandırılmıştır.
Daha sonra $n$-boyutlu Öklid uzayında genelleştirilmiş regle yüzeylerin kesit eğrilikleri (Frank ve Giering, 1979) tarafından çalışılmış ve bu çalışmada genelleştirilmiş regle yüzeyin 2-boyutlu asli regle yüzeylerinin kesit eğriliği Lamarle formülünün genel formu olarak elde edilmiştir. 
$\mathbb{R}_{1}^{n}, \quad n$-boyutlu Minkowski uzayında spacelike ve timelike doğrultman uzaylı genelleştirilmiş timelike regle yüzeyler (Tosun ve Kuruoğlu, 1998; Aydemir ve Kuruoğlu, 2000; 2002) çalışmalarında detaylı biçimde incelenmiş ve genelleştirilmiş timelike regle yüzeyler kesit eğrilikleri de (Ersoy ve Tosun, 2010; 2013) tarafindan araştırılmış ve bu yüzeylerin Lamarle formülleri verilmiştir. Diğer taraftan (Ersoy ve Tosun, 2011) çalışmasında 3-boyutlu Minkowski uzayında 2-boyutlu timelike ve spacelike silindirik olmayan regle yüzeylerin Gauss eğrilikleri ile dağılma parametreleri arasındaki bağıntılar elde edilmiş ve yüzeyin karakterine bağlı olarak üç farklı Lorentzian Lamarle formülü elde edilmiştir. Ayrıca, 3boyutlu Minkowski uzayında pseudo null dayanak eğrili ve null doğrultmanlı açılamaz regle yüzeylerin Lamarle formülü (Öztürk, İlarslan, Koç Öztürk ve Nesovic, 2018) tarafından verilmiştir.

Bu çalışmada, $n$-boyutlu Minkowski uzayında timelike doğrultman uzaylı merkez regle yüzeyli genelleştirilmiş timelike regle yüzeyinin 2-boyutlu timelike ve spacelike asli regle yüzeylerinin kesit eğriliği ile asli dağılma parametreleri arasındaki bağıntılar araştırılarak (Ersoy ve Tosun, 2011) tarafindan elde edilen bulgular genelleştirilecektir.

\section{Timelike Doğrultman Uzaylı Genelleştirilmiş Timelike Regle Yüzeyler}

$\mathbb{R}_{1}^{n}, \quad n$-boyutlu Minkowski uzay1 $\mathbf{x}=\left(x_{1}, x_{2}, \ldots, x_{n}\right)$ ve $\mathbf{y}=\left(y_{1}, y_{2}, \ldots, y_{n}\right) \in \mathbb{R}^{n}$ olmak üzere

$$
\langle\mathbf{x}, \mathbf{y}\rangle=x_{1} y_{1}+x_{2} y_{2}+\ldots+x_{n-1} y_{n-1}-x_{n} y_{n}
$$

Eğer $\langle\mathbf{x}, \mathbf{x}\rangle>0$ veya $\mathbf{x}=\mathbf{0}$ ise $\mathbf{x}$ spacelike vektördür, $\langle\mathbf{x}, \mathbf{x}\rangle<0$ ise $\mathbf{x}$ timelike vektördür ve $\langle\mathbf{x}, \mathbf{x}\rangle=0$ ve $\mathbf{x} \neq \mathbf{0}$ ise $\mathbf{x}$ null (lightlike) vektördür.

Herhangi $\quad \alpha=\alpha(t) \subset \mathbb{R}_{1}^{n} \quad$ eğrisinin $\quad$ hrz vektörü, sırasıyla, spacelike, timelike veya null (lightlike) vektör ise $\alpha$ eğrisi spacelike, timelike veya null (lightlike) eğridir (O’Neill, 1983).

$\mathbb{R}_{1}^{n}, \quad n$-boyutlu Minkowski uzayında $(k+1)$-boyutlu timelike bir regle yüzey parametrik olarak

$$
\varphi\left(t, u_{1}, \ldots, u_{k}\right)=\alpha(t)+\sum_{i=1}^{k} u_{i} e_{i}(t)
$$

ile verilir ve $M$ olarak gösterilmiştir. Burada, $\alpha$ dayanak eğrisi spacelike bir eğri ve $E_{k}(t)=S p\left\{e_{1}(t), \ldots, e_{k}(t)\right\} \quad$ doğrultman uzayı timelike bir altuzaydır (Aydemir ve Kuruoğlu, 2002)

$$
A(t)=S p\left\{e_{1}(t), \ldots, e_{k}(t), \dot{e}_{1}(t), \ldots, \dot{e}_{k}(t)\right\}
$$

altuzayına $M$ regle yüzeyinin $E_{k}(t)$ doğrultman uzayına göre asimptotik demeti adını alır. $A(t)$ timelike bir altuzaydır. Eğer $\operatorname{boy} A(t)=k+m, \quad 0 \leq m \leq k \quad$ ise $\quad A(t)$ asimptotik demetinin doğrultman uzayı ihtiva eden $\quad\left\{e_{1}(t), \ldots, e_{k}(t), a_{k+1}(t), \ldots, a_{k+m}(t)\right\}$ olacak şekilde bir ortonormal bazı bulunabilir. Böylece

$$
\begin{gathered}
\varepsilon_{\mu} \alpha_{v \mu}=-\varepsilon_{v} \alpha_{\mu v}, \quad \varepsilon_{v}=\left\langle e_{v}, e_{v}\right\rangle= \pm 1 \\
\kappa_{1}>\kappa_{2}>\ldots>\kappa_{m}>0
\end{gathered}
$$

olmak üzere $\left\{e_{1}(t), \ldots, e_{k}(t)\right\}$ ortonormal bazı için

Lorentz metriği ile verilmiştir. 


$$
\begin{aligned}
& \dot{e}_{\sigma}=\sum_{\mu=1}^{k} \alpha_{\sigma \mu} e_{\mu}+\kappa_{\sigma} a_{k+\sigma}, 1 \leq \sigma \leq m \\
& \dot{e}_{m+\rho}=\sum_{\mu=1}^{k} \alpha_{(m+\rho) \mu} e_{\mu} \quad, 1 \leq \rho \leq k-m
\end{aligned}
$$

bağıntıları geçerlidir.

\section{Ayrica}

$$
T(t)=S p\left\{e_{1}(t), \ldots, e_{k}(t), \dot{e}_{1}(t), \ldots, \dot{e}_{k}(t), \dot{\alpha}(t)\right\}
$$

altuzayına $M$ regle yüzeyinin $E_{k}(t)$ doğrultman uzayına göre teğetsel demeti denir. $k+m \leq \operatorname{boy} T(t) \leq k+m+1,0 \leq m \leq k$ sağlanır. Bu iki durumu ayrı ayrı incelenir. Eğer $\operatorname{boy} T(t)=k+m$ ise bu takdirde $\left\{e_{1}(t), \ldots, e_{k}(t), a_{k+1}(t), \ldots, a_{k+m}(t)\right\} \quad$ hem asimptotik hem de teğetsel demetin bir bazıdır.

Eğer boy $T(t)=k+m+1$ ise $T(t)$ 'nin

$$
\left\{e_{1}(t), \ldots, e_{k}(t), a_{k+1}(t), \ldots, a_{k+m}(t), a_{k+m+1}(t)\right\}
$$

şeklinde bir ortonormal bazı bulunabilir. Her iki durumda da $T(t)$ teğetsel demeti bir timelike altuzaydır (Aydemir ve Kuruoğlu, 2002).

$\mathrm{Bu}$ çalışmada $\operatorname{boy} T(t)=k+m+1 \quad$ kabul edilecektir çünkü bu durumda $(k+1)-$ boyutlu timelike doğrultman uzaylı timelike regle yüzeyi $M$ 'nin merkez uzayı adı verilen ve $Z_{k-m}(t)$ ile gösterilen $(k-m)$-boyutlu bir altuzay vardır. $Z_{k-m}(t) \subset E_{k}(t)$ altuzayı spacelike veya timelike bir altuzaydır.

$M$ 'nin $\alpha$ dayanak eğrisi boyunca $Z_{k-m}(t)$ uzayını doğrultman uzayı olarak hareket ettirerek $M$ tarafindan ihtiva edilen $(k-m+1)$-boyutlu bir regle yüzey elde edilir. Bu yüzey $\Omega$ ile gösterilir ve merkez regle yüzey olarak adlandırılır. $Z_{k-m}(t)$ doğrultman uzayı spacelike (veya timelike) ise $\Omega$ merkez regle yüzey spacelike (veya timelike) regle yüzeydir. $\Omega$ merkez regle yüzeyinin merkez noktalarında $M$ 'nin tanjant uzayları $A(t)$ asimptotik demetine diktir. Bu durumda (1) denkleminde $u_{\sigma}=0$, $1 \leq \sigma \leq m$ olur ve bu merkez regle yüzeyin $\alpha$ dayanak eğrisi için

$$
\dot{\alpha}(t)=\sum_{v=1}^{k} \zeta_{v} e_{v}+\eta_{m+1} a_{k+m+1} \quad \eta_{m+1} \neq 0
$$

bağıntısı vardır (Aydemir ve Kuruoğlu 2000). $M,(k+1)$-boyutlu timelike regle yüzeyinin $\alpha$ spacelike dayanak eğrisi (3) hız vektörüne sahip olmak üzere $\eta_{m+1} \neq 0$ için

$$
P_{\sigma}=\frac{\eta_{m+1}}{\kappa_{\sigma}}, 1 \leq \sigma \leq m
$$

$M$ 'nin $\sigma$. asli dağılma parametresi olarak adlandırılır (Aydemir ve Kuruoğlu 2000).

$n$-boyutlu Minkowski uzayında $M$ timelike doğrultman uzaylı timelike regle yüzeyinin dayanak eğrisi aynı zamanda $M$ 'nin $\Omega$ merkez regle yüzeyinin de dayanak eğrisi iken her $\xi\left(t, u_{v}\right)$ noktasında $\eta_{m+1} \neq 0$ olmak üzere $M$ 'nin $E_{k}(t)$ doğrultman uzayına ortogonal olan bir normal vektör alanı

$$
\mathbf{n}=\sum_{\sigma=1}^{m} u_{\sigma} \kappa_{\sigma}(t) a_{k+\sigma}(t)+\eta_{m+1} a_{k+m+1}(t),
$$

şeklinde tanımlanır, öyle ki bu normal vektör alanı, timelike doğrultman uzayına ortogonal olduğundan daima spacelike vektör alanıdır. 
Alışılagelmiş indeks yazım şeklini kullanmak

için $u_{0}=t$ alınırsa $\varepsilon_{v}=\left\langle e_{v}, e_{v}\right\rangle= \pm 1$ olmak

üzere birinci temel formun katsayıları

$$
\begin{array}{ll}
g_{00}=-g+\sum_{v=1}^{k} \varepsilon_{v}\left(\zeta_{v}+\sum_{\mu=1}^{k} \alpha_{v \mu} u_{\mu}\right)^{2} & \\
g_{v 0}=\varepsilon_{v}\left(\zeta_{v}+\sum_{\mu=1}^{k} \alpha_{v \mu} u_{\mu}\right) & , 1 \leq v \leq k \\
g_{v \mu}=\varepsilon_{v} \delta_{v \mu} & , 0 \leq v, \mu \leq k \\
g=\operatorname{det}\left[g_{i j}\right]=-\sum_{\sigma=1}^{m}\left(u_{\sigma} \kappa_{\sigma}\right)^{2}-\eta_{m+1}^{2} & , 0 \leq i, j \leq k
\end{array}
$$

olarak elde edilir (Ersoy ve Tosun, 2013). Burada $-\sum_{\sigma=1}^{m}\left(u_{\sigma} \kappa_{\sigma}\right)^{2}-\eta_{m+1}^{2} \neq 0$ olduğundan $\left[g_{i j}\right]$ regüler matristir. $\left[g_{i j}\right]$ matrisinin $\left[g^{i j}\right]$ ters matrisinin elemanları

$$
\begin{aligned}
& g^{00}=-g^{-1} \\
& g^{\nu 0}=\left(\zeta_{v}+\sum_{\mu=1}^{k} \alpha_{v \mu} u_{\mu}\right) g^{-1} \quad, 1 \leq v \leq k \\
& g^{\nu \lambda}=\left(\varepsilon_{\nu} \delta_{\nu \lambda} g-\left(\zeta_{v}+\sum_{\mu=1}^{k} \alpha_{\nu \mu} u_{\mu}\right)\left(\zeta_{\lambda}+\sum_{\mu=1}^{k} \alpha_{\lambda \mu} u_{\mu}\right)\right) g^{-1} \quad, \quad 1 \leq \nu, \lambda \leq k
\end{aligned}
$$

olarak bulunur. (5) ve (6) eşitlikleri (Blaschke, 1945) tarafından verilen Koszul eşitliğinde yerine yazılırsa Christoffel sembolleri $1 \leq v, \mu, \lambda \leq k$ için;

$$
\begin{aligned}
\Gamma_{00}^{0}=\frac{1}{2 g}\left[\frac{\partial g}{\partial u_{0}}+\sum_{v=1}^{k}\left(\zeta_{v}+\sum_{\mu=1}^{k} \alpha_{v \mu} u_{\mu}\right) \frac{\partial g}{\partial u_{v}}\right], \\
\Gamma_{00}^{\lambda}=\frac{1}{2 g}\left[-\left(\zeta_{\lambda}+\sum_{\mu=1}^{k} \alpha_{\lambda \mu} u_{\mu}\right)\left(\frac{\partial g}{\partial u_{0}}+\sum_{v=1}^{k}\left(\zeta_{v}+\sum_{\mu=1}^{k} \alpha_{\nu \mu} u_{\mu}\right) \frac{\partial g}{\partial u_{v}}\right)\right. \\
\left.+2 g\left(\left(\dot{\zeta}_{\lambda}+\sum_{\mu=1}^{k} \dot{\alpha}_{\lambda \mu} u_{\mu}\right)+\sum_{v=1}^{k}\left(\zeta_{v}+\sum_{\mu=1}^{k} \alpha_{v \mu} u_{\mu}\right) \alpha_{\lambda v}+\frac{1}{2} \varepsilon_{\lambda} \frac{\partial g}{\partial u_{\lambda}}\right)\right], \\
\Gamma_{v \mu}^{0}=\Gamma_{\mu \nu}^{0}=0, \\
\Gamma_{v \mu}^{\lambda}=\Gamma_{\mu v}^{\lambda}=0, \\
\Gamma_{\lambda 0}^{0}=\Gamma_{0 \lambda}^{0}=\frac{1}{2 g} \frac{\partial g}{\partial u_{\lambda}}, \\
\Gamma_{\nu 0}^{\lambda}=\Gamma_{0 v}^{\lambda}=\frac{1}{2 g}\left[-\left(\zeta_{\lambda}+\sum_{\mu=1}^{k} \alpha_{\lambda \mu} u_{\mu}\right) \frac{\partial g}{\partial u_{v}}+2 g\left(\alpha_{\lambda v}\right)\right] .
\end{aligned}
$$


şekilde elde edilir (Ersoy ve Tosun, 2013).

$M ' \operatorname{nin}\left\{u_{0}, u_{1}, \ldots, u_{k}\right\}$ koordinat sisteminin koordinat komşuluğunda tanjant uzayının bir tabanı $\left\{\partial / \partial u_{i}=\partial_{i} \mid 0 \leq i \leq k\right\}$ ile gösterilmek üzere $M$ 'nin Riemann-Christoffel eğrilik tensörü

$$
R_{h l i j}=\sum_{r=0}^{k} g_{r h}\left(\frac{\partial}{\partial u_{i}} \Gamma_{j l}^{r}-\frac{\partial}{\partial u_{j}} \Gamma_{i l}^{r}-\sum_{s=0}^{k} \Gamma_{i l}^{s} \Gamma_{j s}^{r}+\sum_{s=0}^{k} \Gamma_{j l}^{s} \Gamma_{i s}^{r}\right)
$$

olarak verilir. (Blaschke, 1945).

Böylece (7)'de verilen bağıntılar son denklemde yerine yazilarak $R_{i j 00}, R_{i j \nu \mu}$ ve
$R_{v 0 \mu 0} \quad$ eğrilikleri $\quad M$ 'nin birinci temel formunun determinant1 $g$ ve $g$ 'nin birinci ve ikinci mertebeden kısmi türevleri cinsinden

$$
\begin{array}{ll}
R_{i j 00}=0 & , 0 \leq i, j \leq k \\
R_{i j \nu \mu}=0 & , 0 \leq i, j \leq k, 1 \leq v, \mu \leq k \\
R_{\nu 0 \mu 0}=\frac{1}{2} \frac{\partial^{2} g}{\partial u_{v} \partial u_{\mu}}-\frac{1}{4 g} \frac{\partial g}{\partial u_{v}} \frac{\partial g}{\partial u_{\mu}} & , 1 \leq v, \mu \leq k
\end{array}
$$

olarak bulunur (Ersoy ve Tosun, 2013).

\section{3. -Boyutlu Minkowski Uzayında Asli Regle Yüzeylerin Kesit Eğrilikleri}

$\mathbb{R}_{1}^{n}, \quad n$-boyutlu Minkowski uzayında $(k+1)$-boyutlu $M$ timelike regle yüzeyinin $\alpha$ dayanak eğrisinin $\zeta \in Z_{k-m}(t)$ noktasındaki her bir $h_{\sigma}=S p\left\{e_{\sigma}\right\}, 1 \leq \sigma \leq m$ uzayı $E_{k}(t)$ içindedir.

$\zeta+u e_{\sigma}(t)$ parametrik ifadesi ile verilen ve asli 1şınlar olarak adlandırılan $h_{\sigma}$ 1-boyutlu uzayları $Z_{k-m}(t)$ merkez uzayına total olarak ortogonal olur.

$\Omega$ merkez regle yüzeyinin $\alpha$ spacelike dayanak eğrisi boyunca hareket eden $h_{\sigma}=\operatorname{Sp}\left\{e_{\sigma}\right\}, \quad 1 \leq \sigma \leq m$, asli 1şınları 2boyutlu regle yüzeyler oluştururlar.
Bu regle yüzeylere $M$ 'nin asli regle yüzeyleri denir ve $M_{\sigma}$ ile gösterilen asli regle yüzeyler parametrik olarak $(t, u) \in(I, \mathbb{R})$ ve $1 \leq \sigma \leq m$ olmak üzere

$$
\varphi_{\sigma}(t, u)=\alpha(t)+u e_{\sigma}(t)
$$

parametrik ifadesi ile verilir.

$\Omega$ merkez regle yüzeyli genelleştirilmiş timelike regle yüzeyi $M$ 'nin ortogonal yörüngesi $\Omega$ merkez regle yüzeyinin $\alpha$ dayanak eğrisi ise $\alpha, M_{\sigma}$ asli regle yüzeyinin striksiyon çizgisi ile çakışır.

$\mathbb{R}_{1}^{n}, \quad n$-boyutlu Minkowski uzayında timelike doğrultman uzaylı, merkez regle yüzeyli genelleştirilmiş timelike regle yüzeyin $\Omega$ merkez regle yüzeyi timelike ya da spacelike olabilir. Eğer $\Omega$ merkez regle yüzeyi timelike ise $h_{\sigma}, \quad 1 \leq \sigma \leq m$, doğrultmanları spacelike olur. 
Böylece $\quad M$ 'nin spacelike ortogonal yörüngesi boyunca $h_{\sigma}$ doğrultmanlarının hareketiyle oluşan $m$ tane spacelike striksiyon eğrili asli regle yüzeyi oluşur.

Diğer taraftan $\Omega$ merkez regle yüzeyi spacelike iken $h_{\sigma}, \quad 1 \leq \sigma \leq m$, doğrultmanlarından bir tanesi timelike ve diğer $(m-1)$ tanesi spacelike olur. Böylece merkez regle yüzeyli genelleştirilmiş timelike regle yüzeyin spacelike ortogonal yörüngesi boyunca $h_{\sigma}$ doğrultmanlarının hareketiyle oluşan striksiyon eğrili asli regle yüzeylerinden bir tanesi timelike ve $(m-1)$ tanesi spacelike olur. Sonuç olarak spacelike asli regle yüzeylerin ve timelike doğrultmanlı timelike asli regle yüzeyin kesit eğrilikleri ayrı ayrı incelemek üzere aşağıdaki teoremler verilebilir.

Teorem 3.1. $\mathbb{R}_{1}^{n}, \quad n$-boyutlu Minkowski uzayında timelike doğrultman uzayl, merkez regle yüzeyli genelleştirilmiş timelike regle yüzey $M$ 'nin 2-boyutlu timelike doğrultmanl timelike asli yüzeyi $M_{s}, \quad 1 \leq s \leq m$ olsun. $\zeta \in \Omega \subset M$ ve $u \in \mathbb{R}$ olmak üzere $M_{s}$, s. timelike asli regle yüzeyinin $h_{s}=S p\left\{e_{s}\right\}$ doğrultmanı üzerinde bir $\zeta+u e_{s}$ noktasındaki kesit ĕgriliği

$$
K_{\zeta+u e_{s}}\left(e_{s}, \mathbf{n}\right)=\frac{P_{s}^{2}}{\left(u^{2}+P_{s}^{2}\right)^{2}}
$$

olur, burada $P_{s}, 1 \leq s \leq m, M$ 'nin $s$. asli dă̆llma parametresidir.

İspat. $\mathbb{R}_{1}^{n}, n$-boyutlu Minkowski uzayında $M$ 'nin ortogonal yörüngesi boyunca $1 \leq s \leq m$ olmak üzere $h_{s}=S p\left\{e_{s}\right\}$ timelike asli 1şının oluşturduğu 2-boyutlu timelike doğrultmanlı timelike asli regle yüzeyi $M_{s}$

$$
\varphi_{s}(t, u)=\alpha(t)+u e_{s}(t)
$$

parametrizasyonu ile verilir. $M_{s}$ 'nin birinci temel formunun matrisinin determinant1

$$
g=-\left(u \kappa_{s}\right)^{2}-\eta_{m+1}^{2}
$$

olur. Böylece, g 'nin birinci ve ikinci mertebeden kısmi türevleri

$$
\frac{\partial g}{\partial u}=-2 u \kappa_{s}^{2} \quad, \frac{\partial^{2} g}{\partial u^{2}}=-2 \kappa_{s}^{2}
$$

olarak bulunur. Burada (9) yardımıyla $M_{s}$, $1 \leq s \leq m$, 2-boyutlu timelike asli yüzeyinin Riemann-Christoffel eğriliği

$$
R_{s 0 s 0}=-\frac{1}{2} \frac{\partial^{2} g}{\partial u^{2}}+\frac{1}{4 g}\left(\frac{\partial g}{\partial u}\right)^{2}
$$

dir ve (12) denkleminde (11)'de verilen bağıntılar yerine yazılırsa

$$
R_{s 0 s 0}=\frac{-2\left(\kappa_{s}\right)^{2}}{2}-\frac{4\left(u \kappa_{s}\right)^{2}\left(\kappa_{s}\right)^{2}}{4\left(-\left(u \kappa_{s}\right)^{2}-\eta_{m+1}^{2}\right)}
$$

bulunur. n yüzey normal vektör alanı ve $\zeta \in \Omega \subset M \quad$ ve $u \in \mathbb{R}$ olmak üzere $h_{s}=S p\left\{e_{s}\right\}$ doğrultmanı üzerinde $\zeta+u e_{s}$ noktasında $\left(e_{s}, \mathbf{n}\right), \quad 1 \leq s \leq m, \quad$ timelike kesitinin eğriliği

$$
K_{\zeta+u e_{s}}\left(e_{s}, \mathbf{n}\right)=\frac{R_{s 0 s 0}}{\left\langle e_{s}, e_{s}\right\rangle\langle\mathbf{n}, \mathbf{n}\rangle-\left\langle e_{s}, \mathbf{n}\right\rangle^{2}}
$$

ile verilir ve (13) denklemi göz önüne alınırsa 
$K_{\zeta+u e_{s}}\left(e_{s}, \mathbf{n}\right)=\frac{\left(\kappa_{s} \eta_{m+1}\right)^{2}}{\left(u \kappa_{s}\right)^{4}+2\left(u \kappa_{s} \eta_{m+1}\right)^{2}+\left(\eta_{m+1}\right)^{4}}$ elde edilir. Payı ve paydası $\kappa_{s}^{4}$ ile sadeleştirildiğinde

$$
K_{\zeta+u e_{s}}\left(e_{s}, \mathbf{n}\right)=\frac{-\left(\frac{\eta_{m+1}}{\kappa_{s}}\right)^{2}}{u^{4}+2 u^{2}\left(\frac{\eta_{m+1}}{\kappa_{s}}\right)^{2}+\left(\frac{\eta_{m+1}}{\kappa_{s}}\right)^{4}}
$$

bulunur. $\mathrm{Bu}$ son denklemde (4) denklemi yerine yazilırsa

$$
K_{\zeta+u e_{s}}\left(e_{s}, \mathbf{n}\right)=\frac{-P_{s}^{2}}{u^{4}+2 u^{2} P_{s}^{2}+P_{s}^{4}}
$$

elde edilir.

Böylece $M_{s}, 1 \leq s \leq m, 2$-boyutlu timelike doğrultmanlı timelike asli regle yüzeyinin kesit eğriliği olan (10) denklemi bulunur ve ispat tamamlanır.

Sonuç 3.1. $\mathbb{R}_{1}^{n}, \quad n$-boyutlu Minkowski uzayında timelike doğrultman uzayl genelleştirilmiş timelike regle yüzeyin spacelike ortogonal yörüngesi boyunca timelike asli uşının hareketiyle oluşan 2boyutlu timelike asli regle yüzeyinin kesit eğriliği (Ersoy ve Tosun, 2011) tarafindan verilen $\mathbb{R}_{1}^{3}$, 3-boyutlu Minkowski uzayında timelike doğrultmalı timelike yüzeyinin Gauss ĕgriliği ile dă̆lma parametresi arasındaki bağıntı olan Lorentzian Lamarle formülünün genelleştirilmişidir.

Teorem 3.2. $\mathbb{R}_{1}^{n}, n$-boyutlu Minkowski uzayında timelike doğrultman uzayl, merkez regle yüzeyli genelleştirilmiş timelike regle yüzey $M$ 'nin 2-boyutlu $\sigma$. spacelike asli regle yüzeyi $M_{\sigma}, \quad 1 \leq \sigma \leq m$ olsun. $\zeta \in \Omega \subset M \quad$ ve $u \in \mathbb{R}$ için $h_{\sigma}=\operatorname{Sp}\left\{e_{\sigma}\right\}$ spacelike doğrultmanı üzerinde $\zeta+u e_{\sigma}$ noktasında $M_{\sigma} \sigma$. spacelike asli yüzeyinin kesit e $\breve{g r i l i g ̆ i}$

$$
K_{\zeta+u e_{\sigma}}\left(e_{\sigma}, \mathbf{n}\right)=-\frac{P_{\sigma}^{2}}{\left(u^{2}+P_{\sigma}^{2}\right)^{2}}
$$

olur, burada $P_{\sigma}, 1 \leq \sigma \leq m$, $M$ 'nin $\sigma$. asli dă̆llma parametresidir.

İspat. $M$ 'nin spacelike ortogonal yörüngesi boyunca $h_{\sigma}, 1 \leq \sigma \leq m$, spacelike asli ışınının oluşturduğu $\quad M_{\sigma}, \quad 1 \leq \sigma \leq m, \quad$ 2-boyutlu spacelike $\sigma$. asli regle yüzeyleri

$$
\varphi_{\sigma}(t, u)=\alpha(t)+u e_{\sigma}(t) \quad, \quad 1 \leq \sigma \leq m
$$

parametrizasyonu ile verilir. $M_{\sigma} \sigma$. spacelike asli yüzeyinin birinci temel formunun matrisinin determinant $g=-\left(u \kappa_{\sigma}\right)^{2}-\eta_{m+1}^{2}$, $1 \leq \sigma \leq m$, olup $g$ 'nin birinci ve ikinci mertebeden kısmi türevleri

$$
\frac{\partial g}{\partial u}=-2 u \kappa_{\sigma}^{2} \quad, \quad \frac{\partial^{2} g}{\partial u^{2}}=-2 \kappa_{\sigma}^{2}
$$

dır. $\left(e_{\sigma}, \mathbf{n}\right), \quad 1 \leq \sigma \leq m$, spacelike kesitinin eğriliğini bulmak için $\zeta+u e_{\sigma}$ noktasında kesit eğriliği denkleminde son eşitlikler yerine konulur ve gerekli sadeleştirmeler yapılırsa

$$
\begin{aligned}
K_{\zeta+u e_{\sigma}}\left(e_{\sigma}, \mathbf{n}\right) & =\frac{R_{\sigma 0 \sigma 0}}{\left\langle e_{\sigma}, e_{\sigma}\right\rangle\langle\mathbf{n}, \mathbf{n}\rangle-\left\langle e_{\sigma}, \mathbf{n}\right\rangle^{2}} \\
& =-\frac{\left(\kappa_{\sigma} \eta_{m+1}\right)^{2}}{\left(u \kappa_{\sigma}\right)^{4}+2\left(u \kappa_{\sigma} \eta_{m+1}\right)^{2}+\left(\eta_{m+1}\right)^{4}}
\end{aligned}
$$


elde edilir. Bu denklemin payı ve paydası $\kappa_{\sigma}^{4}$ ile sadeleştirilir ve (4) denklemi göz önüne alınırsa

$$
K_{\zeta+u e_{\sigma}}\left(e_{\sigma}, \mathbf{n}\right)=-\frac{P_{\sigma}^{2}}{u^{4}+2 u^{2} P_{\sigma}^{2}+P_{\sigma}^{4}}
$$

elde edilir. Böylece, gerekli düzenlemeler sonucu ispat tamamlanır.

Sonuç 3.2. n-boyutlu Minkowski uzayında timelike doğrultman uzayl genelleştirilmiş timelike regle yüzeyin spacelike ortogonal yörüngesi boyunca, spacelike asli ışılarının hareketiyle oluşan 2-boyutlu spacelike asli regle yüzeylerinin (14) denklemi ile verilen kesit eğriliği (Ersoy ve Tosun, 2011) tarafindan verilen 3-boyutlu Minkowski uzayında 2-boyutlu spacelike regle yüzeylerinin Gauss ĕgriliği ile dă̆llma parametresi arasındaki bağıntı olan Lamarle formülünün genelleştirilmişidir.

Örnek 3.1. $\mathbb{R}_{1}^{5}$, 5-boyutlu Minkowski uzayı $\mathbf{x}=\left(x_{1}, x_{2}, x_{3}, x_{4}, x_{5}\right), \quad \mathbf{y}=\left(y_{1}, y_{2}, y_{3}, y_{4}, y_{5}\right)$ $\in \mathbb{R}^{5}$ olmak üzere

$$
\langle\mathbf{x}, \mathbf{y}\rangle=x_{1} y_{1}+x_{2} y_{2}+x_{3} y_{3}+x_{4} y_{4}-x_{5} y_{5}
$$

Lorentz metriği ile verilsin.

$\mathbb{R}_{1}^{5}$,de $\kappa, \tau$ ve $\delta=\sqrt{\kappa^{2}+\tau^{2}}$ keyfi sabitler olmak üzere $\alpha: I \rightarrow \mathbb{R}_{1}^{5}$ eğrisi

$$
\alpha(t)=\frac{1}{\delta}\left(\begin{array}{l}
2 \tau \delta t \\
\sqrt{3} \kappa \cosh \delta t+\kappa \sinh \delta t+\delta \tau t \\
2 \kappa \delta t \\
\sqrt{3} \tau \cosh \delta t+\tau \sinh \delta t-\kappa \delta t \\
\sqrt{3} \delta \sinh \delta t+\delta \cosh \delta t
\end{array}\right)
$$

olsun. $\langle\dot{\alpha}, \dot{\alpha}\rangle=3 \delta^{2}>0 \quad$ olduğundan $\quad \alpha$ spacelike bir eğridir.

$\alpha$ eğrisinin her noktasında tanımlı $\left\{e_{1}(t), e_{2}(t)\right\}$ ortonormal vektör alan sistemi

$$
\begin{aligned}
& e_{1}(t)=\frac{1}{\delta}\left(\begin{array}{l}
\kappa+\tau \\
\sqrt{3} \kappa \sinh \delta t \\
\kappa-\tau \\
\sqrt{3} \tau \sinh \delta t \\
\sqrt{3} \delta \cosh \delta t
\end{array}\right) \\
& e_{2}(t)=\frac{1}{\sqrt{3} \delta}\left(\begin{array}{l}
\tau-\kappa \\
\kappa \cosh \delta t \\
\kappa+\tau \\
\tau \cosh \delta t \\
\delta \sinh \delta t
\end{array}\right)
\end{aligned}
$$

vektör alanları ile olmak üzere $\left\{e_{1}(t), e_{2}(t)\right\}$ sistemi $\alpha(t) \in \mathbb{R}_{1}^{5}$ noktasında tanjant uzayının 2-boyutlu bir altuzayını gerer. $\mathrm{Bu}$ altuzay $E_{2}(t)$ ile gösterilsin. $\left\langle e_{1}, e_{1}\right\rangle=-1,\left\langle e_{2}, e_{2}\right\rangle=1$ olduğundan $E_{2}(t)$ timelike bir altuzaydır. $\alpha$ dayanak eğrisi boyunca $E_{2}(t)$ doğrultman uzayının hareketiyle oluşan 3-boyutlu timelike regle yüzeyin 2-boyutlu asli regle yüzeylerini göz önüne alalım.

$$
\varphi_{1}(t, u)=\alpha(t)+u e_{1}(t)
$$

parametrizasyonu ile verilen timelike doğrultmanlı timelike asli regle yüzeyi $M_{1}$ olsun. Timelike doğrultmanlı timelike asli 1şın yüzeyi için genelleştirilmiş Lorentzian Lamarle formülünden $M_{1}{ }^{\prime}$ in $\zeta+u e_{\sigma}$ noktasında $\left(e_{1}, n\right)$ timelike kesitinin eğriliği 


$$
K_{\zeta+u e_{1}}\left(e_{1}, n\right)=\frac{2}{\left(2 u^{2}+1\right)^{2}}
$$

bulunur.

$$
\varphi_{2}(t, u)=\alpha(t)+v e_{2}(t)
$$

parametrizasyonu ile verilen spacelike doğrultmanlı spacelike asli ışın yüzeyi $M_{2}$ olsun. Spacelike asli 1şın yüzeyi için genelleştirilmiş Lorentzian Lamarle formülünden $M_{2}$ in $\zeta+u e_{\sigma}$ noktasinda $\left(e_{2}, n\right)$ spacelike kesitinin eğriliği

$$
K_{\zeta+u e_{2}}\left(e_{2}, n\right)=-\frac{6}{\left(2 v^{2}+3\right)^{2}}
$$

elde edilir.

\section{Kaynaklar}

Kruppa, E. (1957). "Analytische und Konstruktive Differentialgeometrie", Wien Springer-Verlag.

Frank H. and Giering, O. 1979. "Zur Schnittkrümmung Verallgemeinerter Regelflachen", Archiv Der Mathematik, Fasc.1, 32, 86-90.

Tosun, M. and Kuruoğlu, N. 1998. "On $(k+1)$-dimensional Time-Like Ruled Surface in the Minkowski Space $\mathbb{R}_{1}^{n}$, J. Inst. Math. Comput. Sci. Math. Ser., 11 (1), 1-9.

Aydemir İ. and Kuruoğlu, N. 2000. "Edge, Center and Principal Ruled Surfaces of $(k+1)$ dimensional Generalized Timelike Ruled Surface in the Minkowski Space $\mathbb{R}_{1}^{n}$, Pure Appl. Math. Sci., 51 (1-2), 19-24.
Aydemir İ. and Kuruoğlu, N. 2002. "Timelike Ruled Surfaces in the Minkowski Space $\mathbb{R}_{1}^{n}$,, Int. J. Appl. Math., 10(2), 149-158.

Ersoy S. and Tosun, M. 2013. "Lorentzian Beltrami-Meusnier Formula", Gen. Math. Notes, 18 (1), 64-87.

Ersoy S. and Tosun, M. 2010. "Sectional Curvature of Timelike Ruled Surface Part I: Lorentzian Beltrami-Euler Formula", Iran. J. Sci. Technol. Trans. A Sci., 34 no.A3, 197214.

Ersoy S. and Tosun, M. 2011. "Lamarle Formula in 3-dimensional Lorentz Space", Math. Commun., 16593-607, (2011).

Öztürk, U. İlarslan, K. Koç Öztürk, E. B. and Nesovic, E. (2018). A Note on Lamarle Formula in Minkowski 3-Space, Tamkang J. of Math., 49(4), 291-300.

O’Neill, B. (1983). "Semi-Riemannian Geometry”, Academic Press, New York.

Blaschke, W. (1945). "Vorlesungen über Differentialgeometrie", 14. Ayfl. Berlin. 